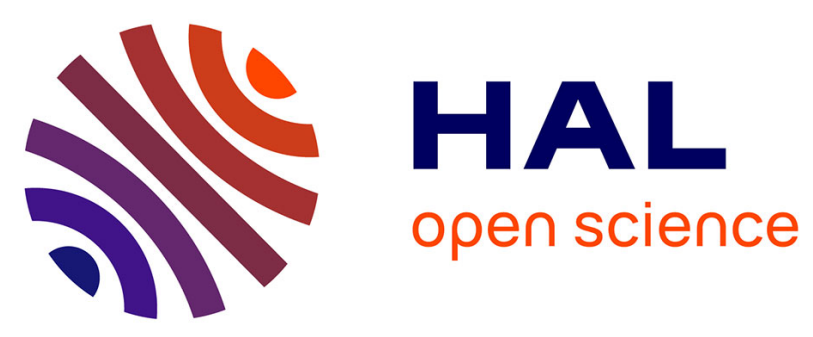

\title{
Comment je fais... pour utiliser le vert d'indocyanine pour contrôler la viabilité des lambeaux en reconstruction vaginale
}

M. Belhadia, F. Narducci, E. Leblanc, Y. Borghesi, M. Boukerrou, B. Hersant

\section{- To cite this version:}

M. Belhadia, F. Narducci, E. Leblanc, Y. Borghesi, M. Boukerrou, et al.. Comment je fais... pour utiliser le vert d'indocyanine pour contrôler la viabilité des lambeaux en reconstruction vaginale. Gynécologie Obstétrique Fertilité \& Sénologie, 2019, 47 (5), pp.484-486. 10.1016/j.gofs.2019.02.009 . inserm-02941542

\section{HAL Id: inserm-02941542 https://www.hal.inserm.fr/inserm-02941542}

Submitted on 26 Oct 2021

HAL is a multi-disciplinary open access archive for the deposit and dissemination of scientific research documents, whether they are published or not. The documents may come from teaching and research institutions in France or abroad, or from public or private research centers.
L'archive ouverte pluridisciplinaire HAL, est destinée au dépôt et à la diffusion de documents scientifiques de niveau recherche, publiés ou non, émanant des établissements d'enseignement et de recherche français ou étrangers, des laboratoires publics ou privés.

\section{(ㅇ)(1) $\$$}

Distributed under a Creative Commons Attribution - NonCommerciall 4.0 International 
Titre : Comment je fais pour utiliser le vert d'indocyanine pour contrôler la viabilité des lambeaux en reconstruction vaginale.

Title: How I do... to use indocyanine green to check the flap viability in vaginal reconstruction.

Titre court : Utilisation du vert d'indocyanine en reconstruction vaginale Short title : Tue use of indocyanine green in vaginal reconstruction

Marine Belhadia ${ }^{1}$, MD, Fabrice Narducci ${ }^{1}$, MD, Eric Leblanc ${ }^{1}$, MD, Yves Borghesi ${ }^{1}$, MD, Malik Boukerrou², MD, PHD, Barbara Hersant ${ }^{1-3}$, MD, PHD.

1 Service de cancérologie gynécologique,

Centre Oscar Lambret,

3 rue Frédéric Combemale, 59000 Lille, France

Tel : 0320295944

Fax : 0320295928

${ }^{2}$ Department of Gynecology and Obstetrics,

University Hospital of South Reunion Island, BP 350 - 97448 Saint Pierre Cedex, Reunion Faculty of Medicine - University of Reunion, 97490 St Denis, Reunion,

CEPOI, Perinatal Center of Study of the Indian Ocean. University Hospital of South Reunion Island, Faculty of medicine, St Pierre 97448, Reunion

Tel : +262692492619

Fax : +262262359114

${ }^{3}$ Department of Plastic and Reconstructive Surgery

Henri Mondor Hospital

51 avenue du Maréchal de Lattre de Tassigny

Créteil 94010, France 
Tel : 0149812111

Fax : 0149812199

Corresponding author : Marine Belhadia, marine.belhadia@hotmail.fr, 0693827442

Conflit d'intérêt : aucun

Mots clés : vert d'indocyanine, reconstruction vaginale, lambeau, DIEP

Keywords : indocyanine green, vaginal reconstruction, flap, DIEP flap 


\section{Introduction:}

L'exentération pelvienne est une chirurgie peu fréquente, lourde et mutilante. Il s'agit d'enlever en monobloc les organes pelviens et a pour indication principale les néoplasies du col utérin. Elle peut être effectuée d'emblée dans les formes localement évoluées, ou après le traitement par radio-chimiothérapie, dans un contexte de récidive pelvienne, ou dans la prise en charge de séquelles liées à la radiothérapie (fistules vésico et/ou recto-vaginales).

La reconstruction immédiate du vagin est un challenge chirurgical. Différentes techniques de reconstruction vaginale existent (1), mais nous nous intéresserons plus particulièrement au deep inferior epigastric perforator (DIEP) vertical pédiculé, (2) décrit pour la première fois en 1989, qui préserve le muscle et l'aponévrose, diminuant ainsi les séquelles pariétales. Cette technique nécessite l'intervention d’un chirurgien plasticien pour effectuer la dissection des perforantes nourricières du lambeau. Le DIEP verticale pédiculé semble une technique de premier choix dans cette indication (3). L'utilisation d'un lambeau dans la reconstruction vaginale permet un comblement pelvien, une protection des structures vasculaires et digestives, une diminution des infections et des fistules en terrain irradié, mais aussi semble améliorer la qualité de vie en maintenant le schéma corporel et en permettant une poursuite de l'activité sexuelle. D’après une étude sur la vie sexuelle des patientes, après reconstruction vaginale suite à une exentération pelvienne, 52\% des patientes ont répondu ne pas avoir repris d'activité sexuelle après la chirurgie, mais 70\% referaient l'intervention comme telle si c'était à refaire (4). 
Une planification préopératoire par imagerie est indispensable. Un angioscanner permettra d'identifier la perméabilité des pédicules épigastriques inférieurs profonds et de visualiser la localisation des principales perforantes musculocutanées, comme nous pouvons le voir sur la figure 1a. Ces perforantes sont contrôlées au doppler, systématiquement, la veille de l'intervention par le chirurgien. Le dessin de la palette cutanée doit être centrée sur ces perforantes.

En peropératoire, la viabilité du lambeau doit être surveiller, cliniquement, avec la couleur, la chaleur et le temps de recoloration du lambeau mais aussi par le doppler, et, une nouvelle technique est disponible pour une analyse plus fine de la viabilité : le vert d'indocyanine. L'enjeu de cette technique est la surveillance de la viabilité pour permettre un changement de stratégie en cas d'échec.

Depuis quelques années le vert d'indocyanine est régulièrement utilisé en pratique chirurgicale et notamment pour la surveillance per opératoire des lambeaux perforants pédiculés et des lambeaux libres (5). C'est un colorant soluble dans l'eau, qui peut être injecté en intraveineux ou en sous cutané. En injection intraveineuse, il permet de marquer la perfusion cutanée, en sous-cutané il permet de cartographier le réseau lymphatique. Son spectre d'absorption est de 805 nm maximum avec réémission à 835 nm. La fluorescence est obtenue grâce à la stimulation du vert par un rayon infrarouge, qui sera détectée par une caméra dédiée, ce qui est la seule limite à son utilisation. Nous utilisons la colonne NOVADAQ®, PINPOINT® Endoscopic Fluorescence Imaging System avec la caméra externe SPY-PHI®. Le vert d'indocyanine se fixe aux protéines plasmatiques, en particuliers les lipoprotéines, ce qui est très utile pour l'utiliser comme marqueur vasculaire. Il possède une demi-vie de 3-4 minutes, et est excrété par les voies biliaires. Il n'a été rapporté que très peu de réactions anaphylactiques (1/42000), mais il reste contre-indiqué chez les patientes allergiques à l'iode. H-existe-de rares effets 
indésirables: nausées, vomissement, bouffées de chaleur, mais qui, du fait de l'anesthésie générale, sont anecdotiques. Il peut être injecté de manière répétée, mais avec une dose maximale de $0,5 \mathrm{ml} / \mathrm{kg}$, comme recommandé par l'ANSM (agence nationale de sécurité du médicament).

\section{La technique : fiche pratique}

En chirurgie gynécologique le vert d'indocyanine peut être utilisé pour repérer le ganglion sentinelle, pour évaluer la viabilité d'un lambeau ou pour évaluer les anastomoses. Dans notre cas nous l'utiliserons pour évaluer la perfusion du lambeau utilisé dans la reconstruction vaginale. Il permettra de repérer et exciser les zones mal perfusées à risque de nécrose.

a) Temps préopératoire

La vascularisation abdominale par les perforantes de l'artère épigastrique est contrôlée préalablement par un angioscanner, avec un repérage des perforantes sur une reconstruction 3D (figure 1a) puis contrôlé au doppler.

b) La palette

L'incision cutanée suit le dessin préétabli (figure 1b), avec un décollement sus aponévrotique de la palette jusqu'aux perforantes, à leurs entrées dans l'aponévrose. La dissection est réalisée par des instruments fins, sous contrôle de loupe de grossissement binoculaire. En illustration, nous avons isolé 3 perforantes de calibres inférieurs à $2 \mathrm{~mm}$ (figure 2), mais après dissection seulement 2 perforantes étaient viables.

c) Pédicule épigastrique inférieur

La dissection est poursuivie le long du pédicule épigastrique inférieur jusqu'à la bifurcation iliaque externe pour permettre une transposition du lambeau plus facile vers le pelvis. Le point de pivot étant cette bifurcation vasculaire. 
d) Contrôle de la viabilité : place du vert d'indocyanine

Une fois le lambeau totalement libéré et pédiculé sur les vaisseaux épigastriques inférieures, nous évaluons cliniquement la viabilité de celui ci par la chaleur, la couleur et le temps de recoloration. Un doppler de contrôle est également effectué. Le contrôle par le vert d'indocyanine est la technique la plus sensible pour cette évaluation (5).

Une dose de $0,2 \mathrm{mg} / \mathrm{kg}$ est injectée en IVD. Nous nous limitons à une dose maximale injectable de $0,5 \mathrm{mg} / \mathrm{kg}$, soit deux injections au maximum par intervention. L'injection doit se faire quand l'équipe chirurgicale est prête. Après quelques secondes, le produit diffuse et permet, à l'aide de la caméra adaptée, d'observer le réseau vasculaire sous cutanée. Un délai de deux minutes est nécessaire pour avoir une diffusion optimale du produit. Nous pouvons voir sur la figure 3, avec deux modes de fluorescence différents (SPY fluorescence et PINPOINT fluorescence), que les $4 \mathrm{~cm}$ à la partie distale supérieure (vers la xiphoïde) du lambeau ne sont pas fluorescents et sont considérés comme mal vascularisés. Dans notre cas nous avons excisé la pointe distale supérieure, qui était cliniquement non viable, et nous avons préservé une partie de la zone suspecte au vert d'indocyanine mais cliniquement viable, à la partie inférieure, pour des raisons de conformation du lambeau. Nous avons pu transposé un lambeau de $14 \mathrm{~cm}$.

e) Conformation du lambeau

Le lambeau est modelé pour permettre d'avoir une cavité vaginale correcte, et un premier temps de suture est effectué voie haute (figure 4).

f) Transposition du lambeau

Puis le lambeau est transposé vers la région périnéale, en arrière du pubis, de façon délicate et sans traction excessive (figure 5). Un opérateur doit faire descendre le lambeau délicatement, en vérifiant l'absence de traction ou torsion du pédicule. Un deuxième opérateur situé du coté de la vulve, va réceptionner le lambeau à l'aide d'une 
pince longuette, dans la cavité pelvienne. En cas de coudure du pédicule sur le péritoine, une section de celui ci peut être réalisée et le pédicule est positionné dans la meilleure conformation possible, le péritoine est ensuite refermé autour par des points séparés de Vicryl. Une suture est ensuite effectuée, au niveau de la nouvelle cavité pelvienne par des points séparés de Vicryl. Un drain permettant des irrigations est laissé en place.

\section{g) Temps postopératoire}

La surveillance du lambeau en reconstruction vaginale est difficile, car l'analyse clinique et au doppler dans le néovagin n'est parfois pas possible au lit de la patiente. L'utilisation d'un endoscope d'ORL peut être utilisé, ou un examen sous sédation au bloc opératoire, peut être nécessaire une à 2 fois par semaine. Une coloration violacée doit faire craindre une souffrance veineuse par compression ou rotation du pédicule.

Dans notre cas, nous avons effectué un contrôle à J9 postopératoire, sous anesthésie générale, à l'aide d'un endoscope (figure 6). Nous avons retrouvé une zone de moins 3 cm d'épidermolyse, au niveau de la paroi antérieure du néovagin, qui correspondait à la zone suspecte au vert d'indocyanine, mais qui n'avait pas été sacrifiée en raison de sa viabilité clinique. Un second examen a été effectué à J14 : la zone d'épidermolyse avait totalement desquamée et était saine. La cicatrisation se poursuivait au niveau des sutures.

\section{Conclusion:}

La réalisation d'un néo vagin dans les suites d'une exentération pelvienne est une intervention peu fréquente et difficile, effectuée chez des patientes fragiles et souvent en terrain irradié. Le vert d'indocyanine permet d'apporter une aide dans la confection du lambeau, pour éviter de laisser en place des zones mal perfusées, à haut risque de nécrose. 
Déclaration d'intérêts : les auteurs ont déclaré n'avoir aucun conflit d'intérêt en lien avec cet article. 
Bibliographie :

1. Ferron G, Martel $P$, Querleu D. Reconstruction vaginale après exentération pelvienne: quand la proposer et quelle technique? Bull Cancer (Paris). 1 mai 2003;90(5):435口40.

2. Binder J-P, Méria P, Desgrandchamps F, Revol M, Servant J-M. Reconstruction vaginale par lambeau perforant basé sur le pédicule épigastrique inférieur après pelvectomie antérieure. Ann Urol. 1 juin 2006;40(3):192 202.

3. Ferron G, Gangloff D, Querleu D, Frigenza M, Torrent JJ, Picaud L, et al. Vaginal reconstruction with pedicled vertical deep inferior epigastric perforator flap (diep) after pelvic exenteration. A consecutive case series. Gynecol Oncol. sept 2015;138(3):603-8.

4. Ratliff CR, Gershenson DM, Morris M, Burke TW, Levenback C, Schover LR, et al. Sexual adjustment of patients undergoing gracilis myocutaneous flap vaginal reconstruction in conjunction with pelvic exenteration. Cancer. 15 nov 1996;78(10):2229035.

5. Echalier C, Pluvy I, Pauchot J. Angiographie au vert d'indocyanine en chirurgie reconstructrice: revue de la littérature. Ann Chir Plast Esthét. 1 déc 2016;61(6):858D67.

\section{Légende des photos:}

\section{Photo 1:}

a) Angioscanner préopératoire, repérage des artères perforantes de l'artère épigastrique inférieure profonde,

b) dessin de la palette cutanée verticale, avec marquage des perforantes

\section{Photo 2:}

Artères perforantes avec un calibre moyen de $2 \mathrm{~mm}$

\section{Photo 3:}

Visualisation du lambeau en lumière blanche, puis au vert d'indocyanine 2 minutes après son injection, en mode PINPOINT fluorescence puis en mode SPY fluorescence

\section{Photo 4:}

Modelage du néovagin avant sa descente vers le pelvis

Photo 5:

Pédicule vasculaire, libre, sans tension excessive

\section{Photo 6:}

Examen sous AG à J10, zone d'épidermolyse de moins de $3 \mathrm{~cm}$ 

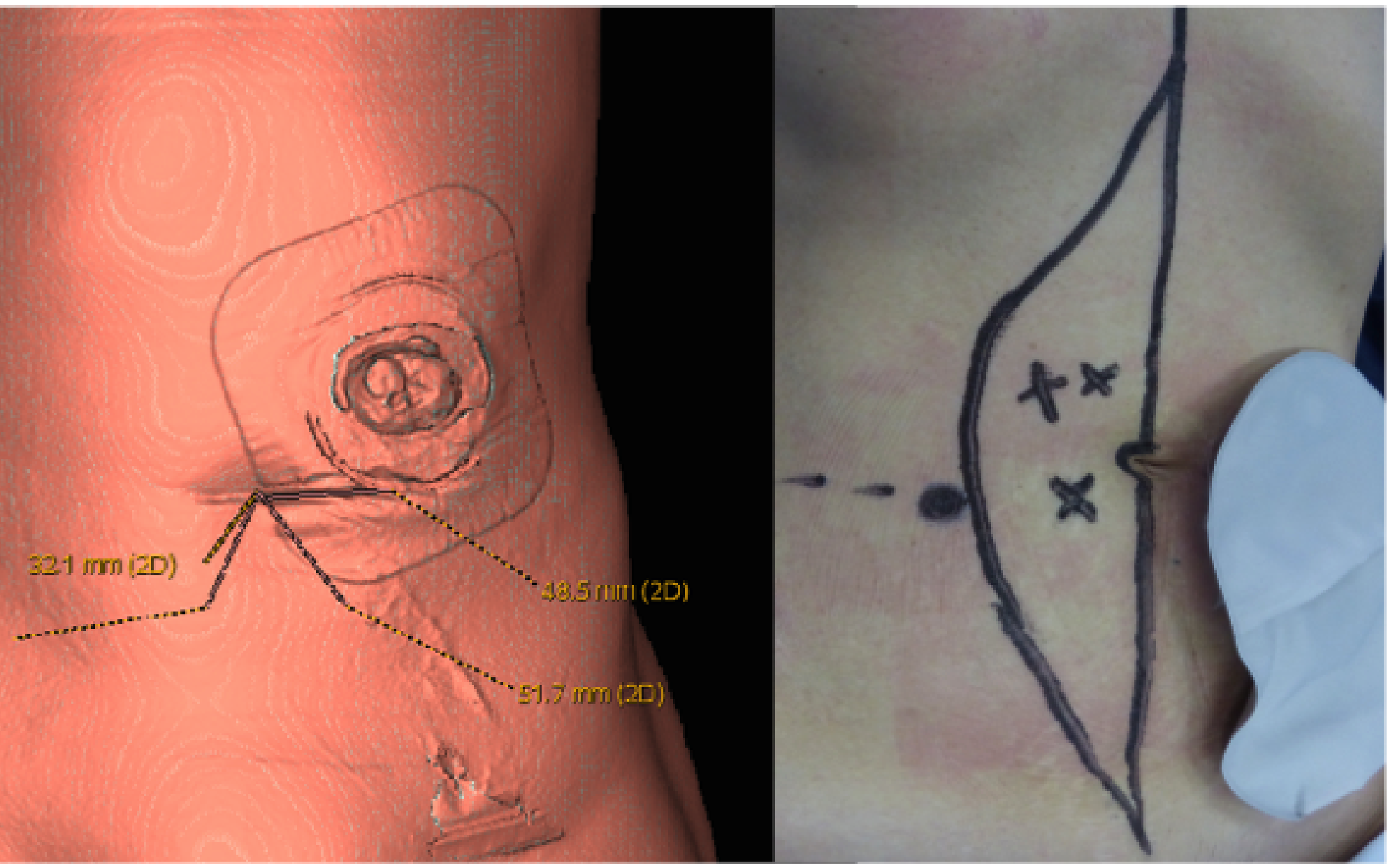


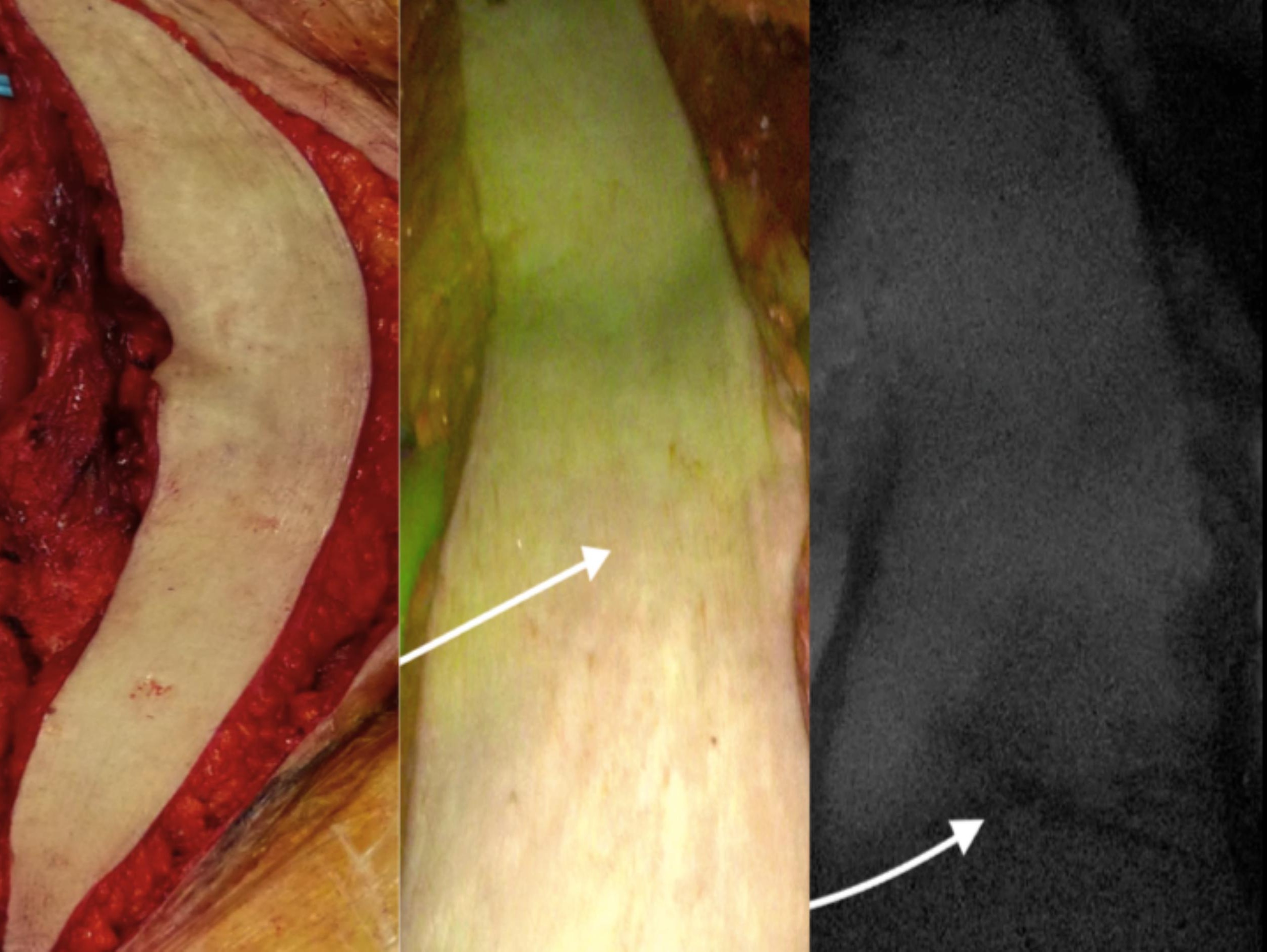




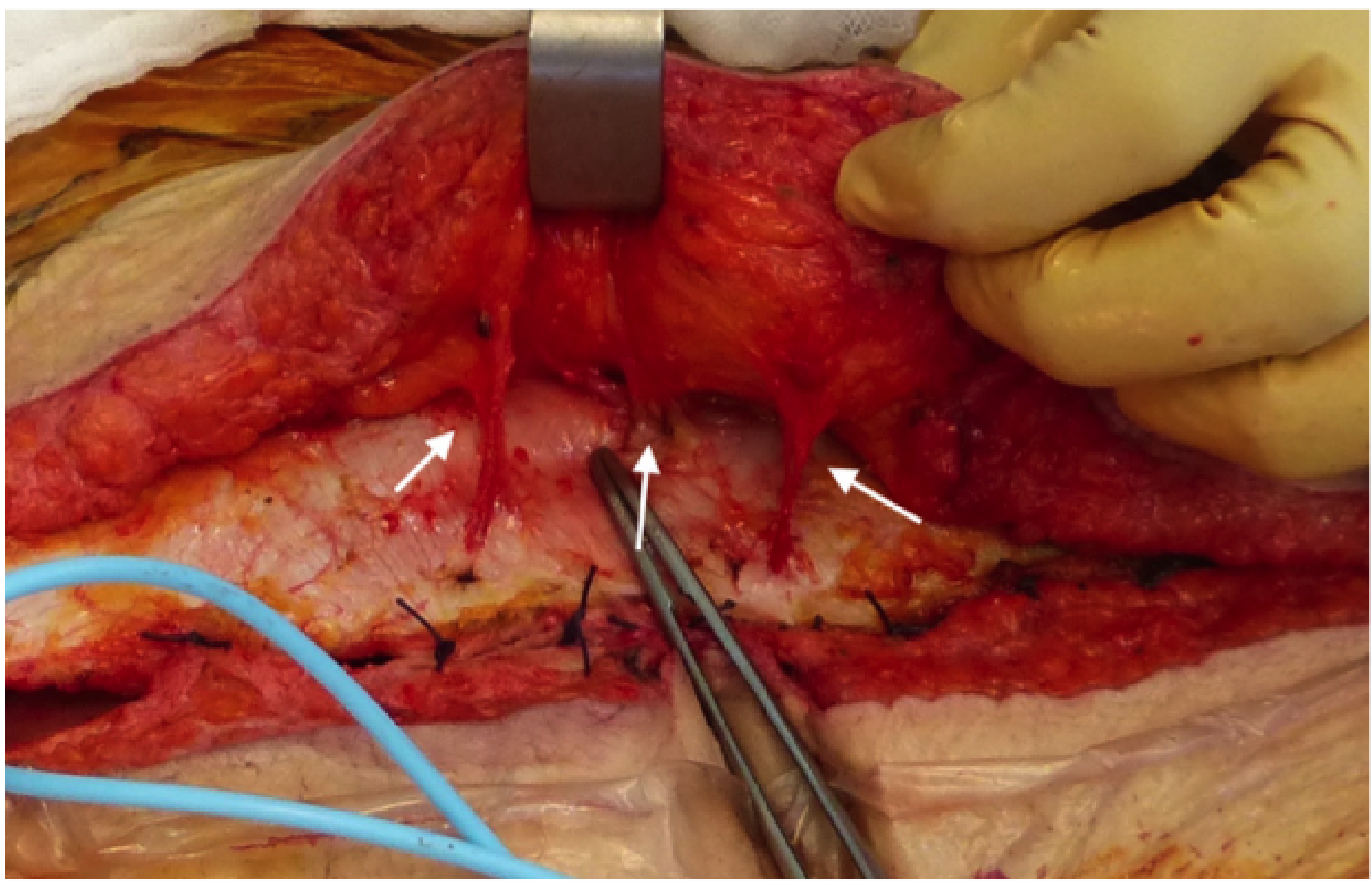




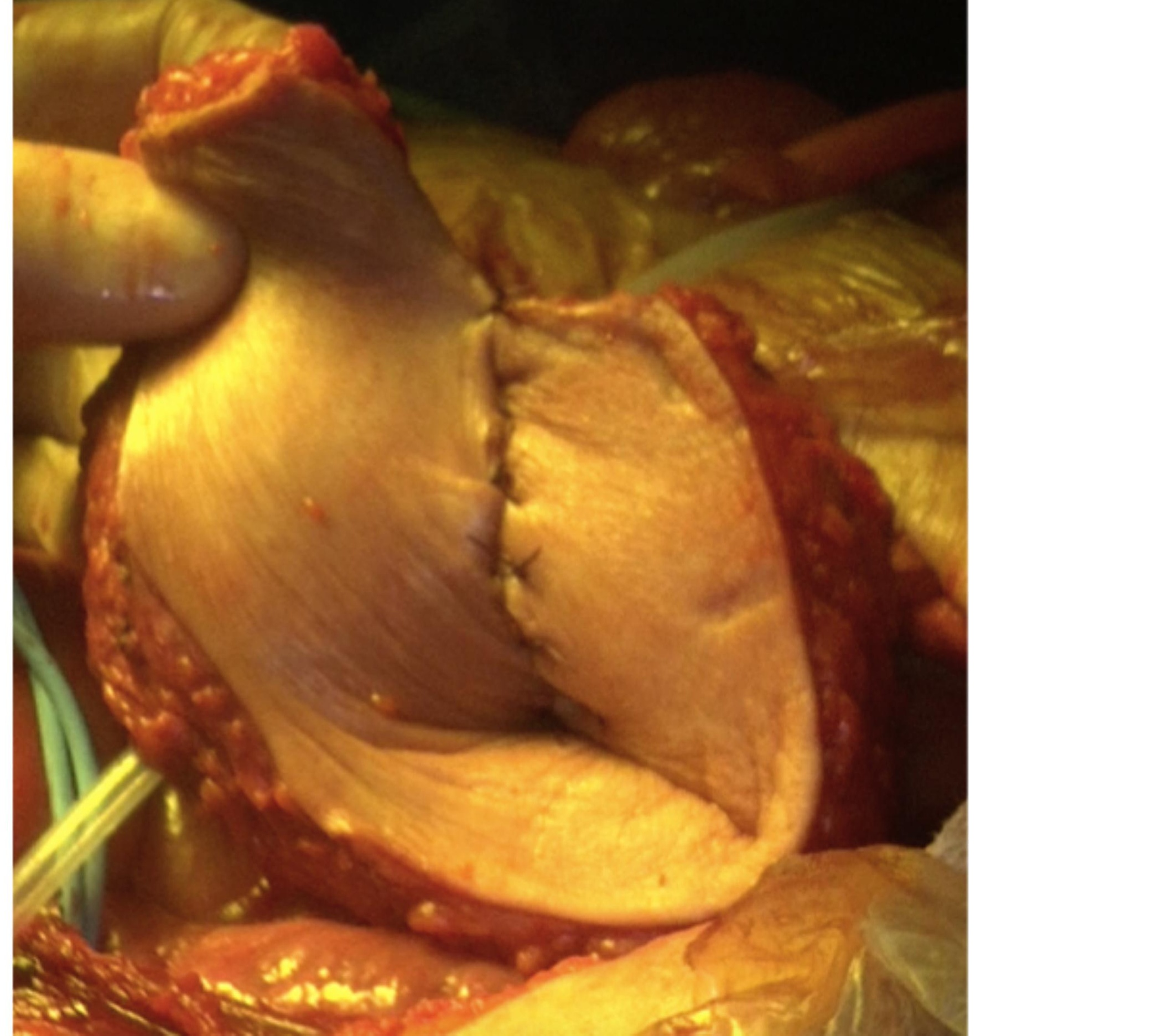




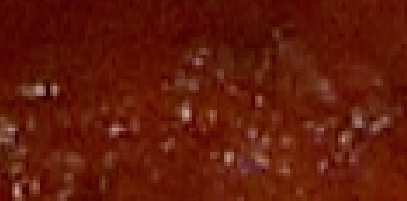

$\because 4$

4. $\quad .64$

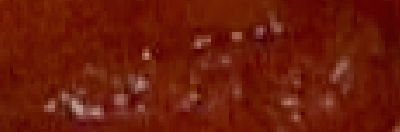

his $\rightarrow+20$ $\therefore$ is 5

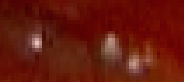

- 4

X.t.

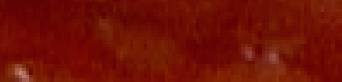

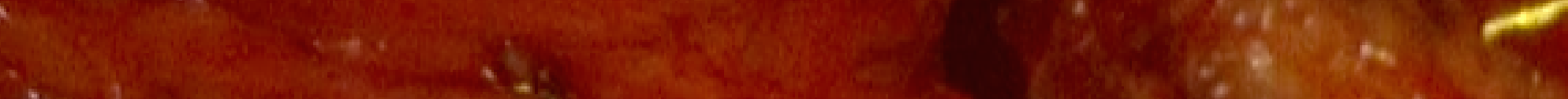

$\therefore+x+2 x+2$

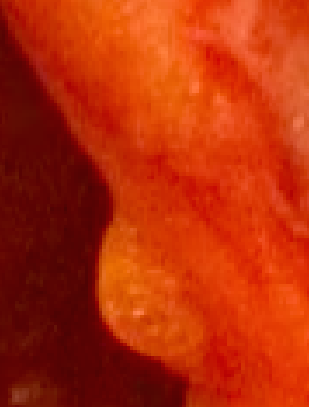

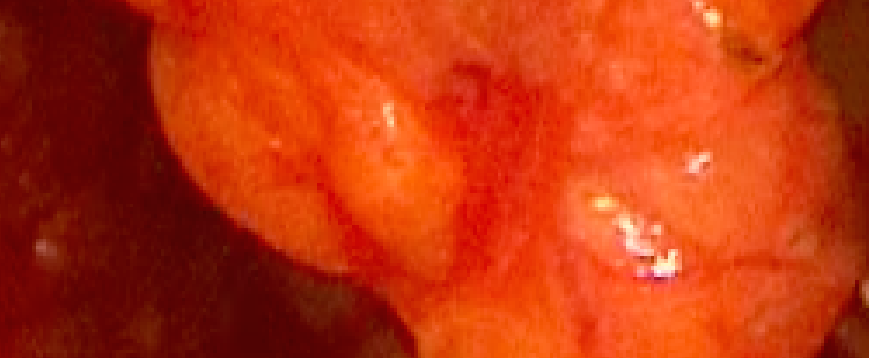
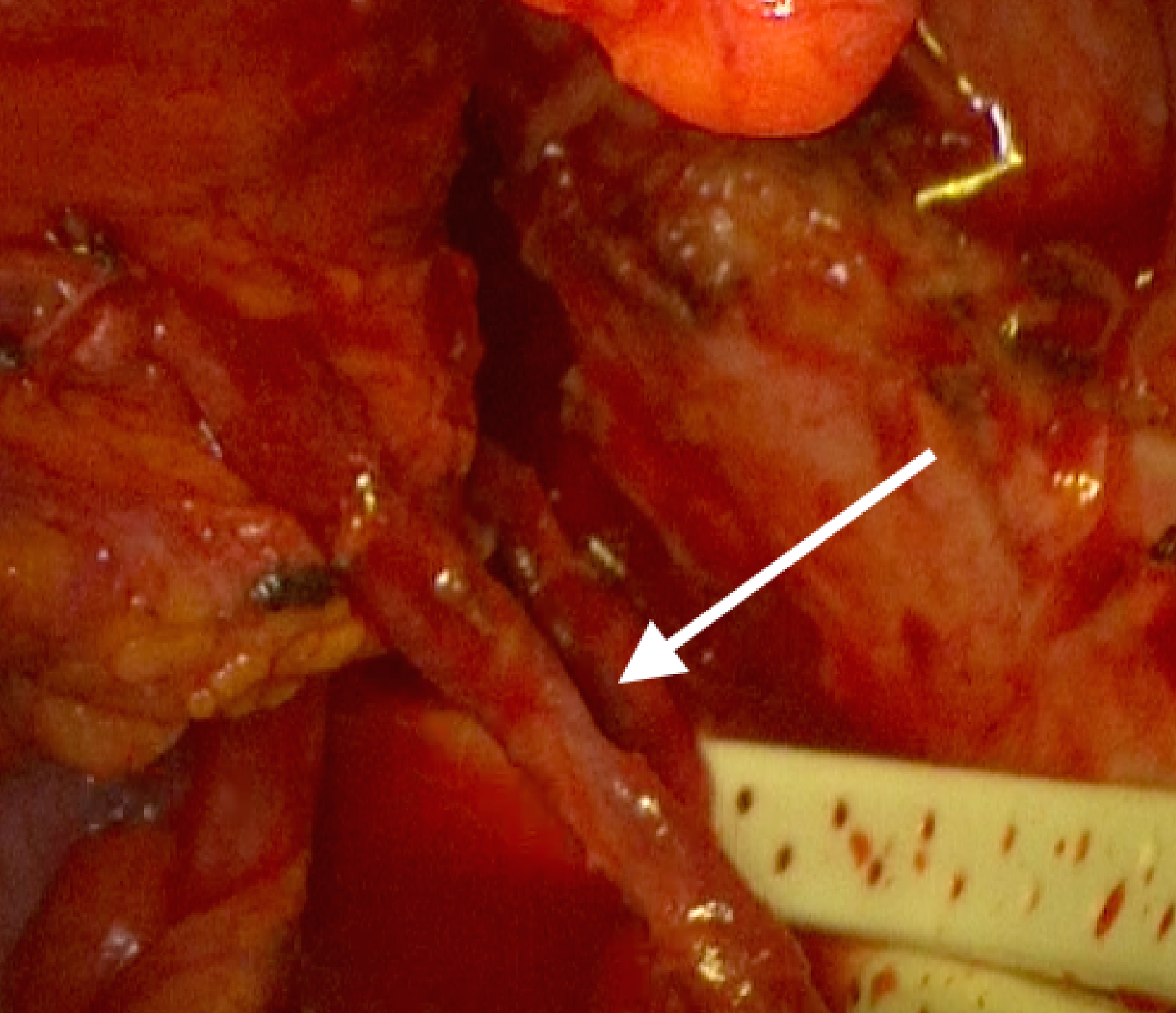

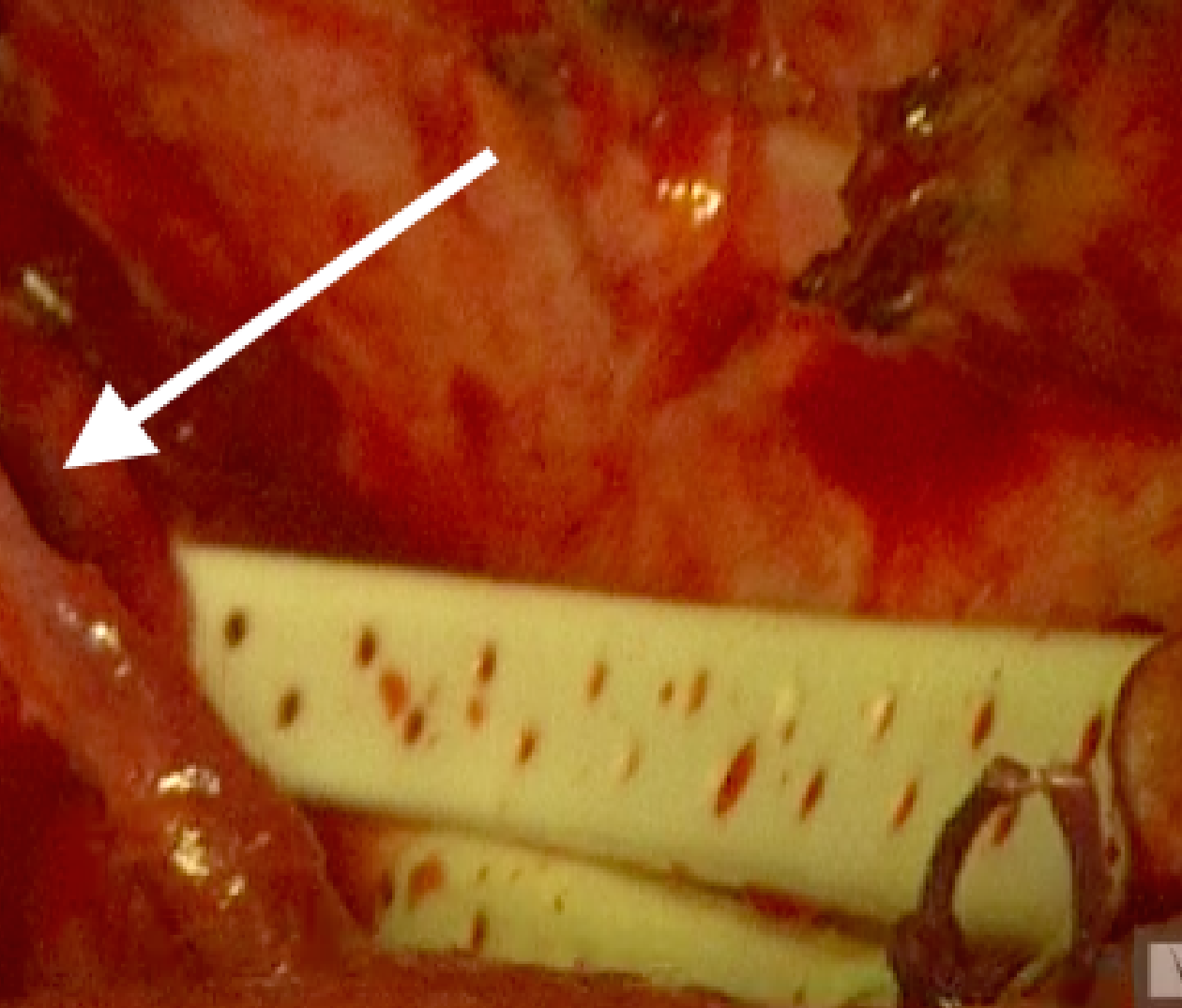

$\triangle N O V \wedge D \wedge Q$ 


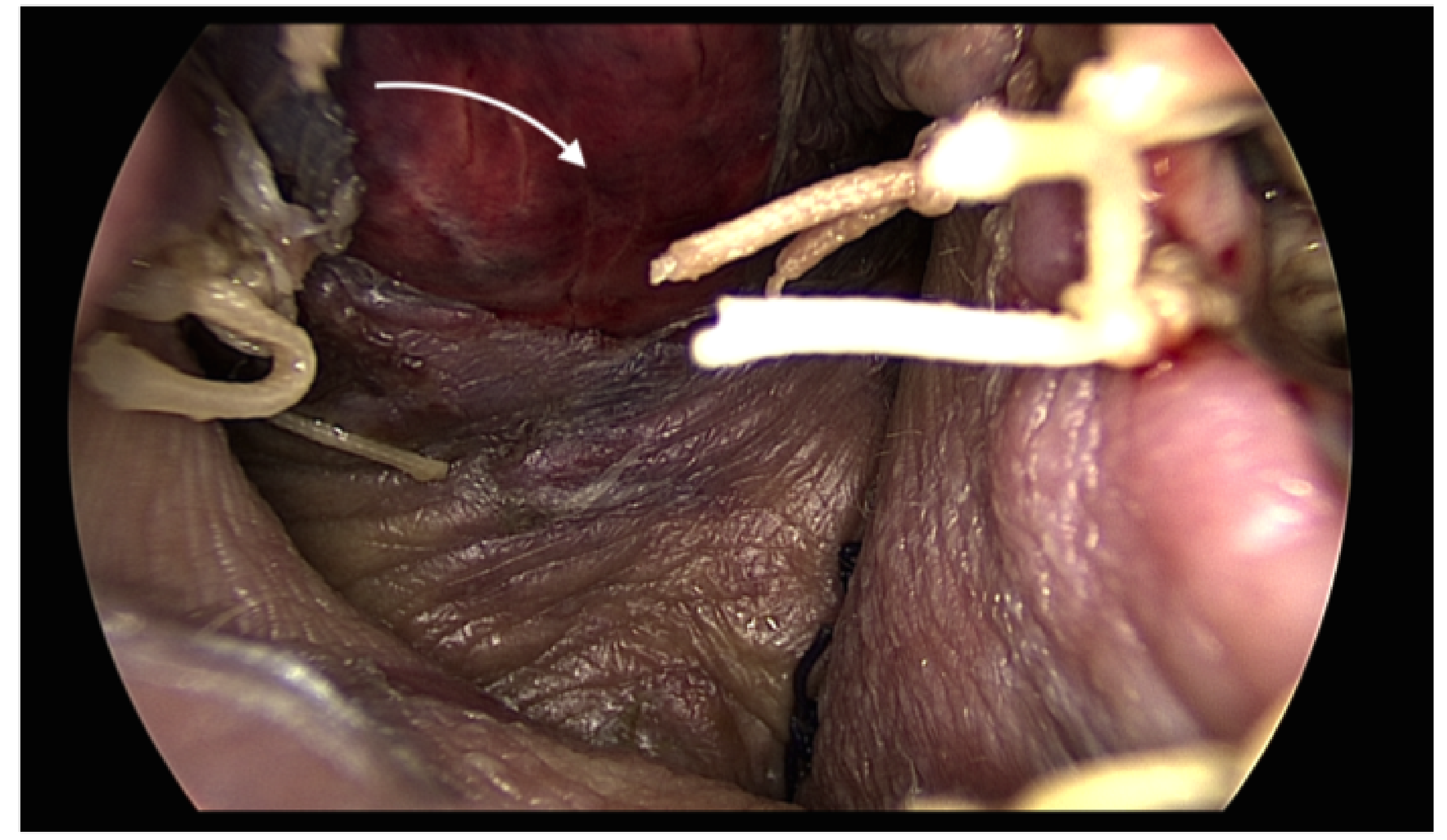

